\title{
Large Intra-Abdominal Abscess Treatment by Marsupialization in 2 Alpacas
}

\author{
Rebecca Rodvold, DVM, Jose L. Mendez-Angulo, DVM, MS, \\ Anna M. Firshman, BVSc, PhD, Diplomate ACVIM and ACVSMR, \\ Erin Malone, DVM, PhD, Diplomate ACVS, and Anne Nicholson, DVM, MS, Diplomate ACVS \\ Department of Veterinary Population Medicine, College of Veterinary Medicine, University of Minnesota, Saint Paul, Minnesota
}

\section{Corresponding Author}

Rebecca Rodvold, DVM, Bucks County Equine, New Hope, PA 18938.

E-mail: rodv0005@umn.edu

Submitted November 2012

Accepted April 2013

DOI:10.1111/j.1532-950X.2014.12271.x
Objectives: To report the clinical signs, diagnostic findings, surgical treatment, postoperative complications, and long-term outcome in 2 adult alpacas with large intraabdominal abscesses treated by marsupialization of the abscess to the ventral body wall. Study Design: Clinical report.

Animals: Alpacas $(\mathrm{n}=2)$.

Methods: Two alpacas each presented with decreased appetite, lethargy, poor body condition, and marked abdominal distension. A complete physical examination, abdominal radiography, and ultrasonography confirmed the presence of large intraabdominal abscesses. Streptococcus equi ssp. zooepidemicus was cultured from the abscess fluid of alpaca 1, and a Gram-negative bacillus from alpaca 2.

Results: Both alpacas had exploratory celiotomy with marsupialization of the abdominal abscess to the ventral body wall. Postoperatively, alpacas were administered systemic antibiotics and non-steroidal anti-inflammatory drugs, and the abscesses were lavaged for several days. Alpaca 2 required a second surgical procedure. The abscesses ultimately resolved in both alpacas, with body wall hernia formation as the only major complication. Both alpacas had good long-term outcomes.

Conclusion: Marsupialization to the ventral abdominal wall with concurrent antibiotic treatment should be considered as a treatment option for alpacas with large intraabdominal abscesses in which complete surgical resection of the abscess is not possible. An abdominal wall hernia can result from this procedure, and clients should be informed of this potential complication before surgery.
Abdominal abscesses are a rare clinical finding in New World Camelids, and we are aware of only 2 reports. ${ }^{1,2}$ A large abdominal abscess was associated with an umbilical infection in a llama cria, ${ }^{1}$ and a liver abscess caused by Corynebacterium pseudotuberculosis was reported in an adult alpaca. ${ }^{2}$ We report the clinical findings, diagnostic procedures, surgical treatment, complications, and long-term outcome of 2 adult alpacas each with a large abdominal abscess of unknown origin. Each alpaca was treated with a different marsupialization technique, both of which were successful.

\section{CLINICAL REPORT}

\section{Alpaca 1}

A 1-year-old female Huacaya alpaca was admitted for evaluation of progressive abdominal distention of 4 months duration. On admission, vital signs were within normal limits and the alpaca was bright, alert, and responsive, with a body condition score (BCS) of $1.5 / 5$ and no history of pyrexia. The abdomen was markedly distended and turgid on palpation, and borborygmi were normal in the cranial third of the abdomen and decreased in the caudal two thirds.

Complete blood cell counts (CBC) and serum biochemical profile were performed. Clinically relevant changes included a mild anemia (PCV 21\%; reference interval: 27-45\%) and decreased serum albumin concentration $(2.3 \mathrm{~g} / \mathrm{dL}$, reference interval: $3.5-4.2 \mathrm{~g} / \mathrm{dL})$. Platelets were increased $\left(1152 \times 10^{3} / \mu \mathrm{L}\right.$, reference interval: $\left.169-692 \times 10^{3} / \mu \mathrm{L}\right)$, as were fibrinogen $(0.4 \mathrm{~g} / \mathrm{dL}$; reference interval: $0.1-0.3 \mathrm{~g} / \mathrm{dL})$ and globulin ( $4.9 \mathrm{~g} / \mathrm{dL}$, reference interval: $2.1-3.1 \mathrm{~g} / \mathrm{dL}$ ) concentrations.

Lateral standing abdominal radiographs showed an extensive ( $\sim 30 \mathrm{~cm}$ diameter) spherical soft tissue mass occupying the mid-caudal abdomen (Fig 1A). The mass caused marked cranioventral displacement of the abdominal viscera. The origin of the mass could not be determined from the radiographs. On abdominal ultrasonography, most of the abdominal organs were obscured by a hyperechoic thick walled structure with contents of mixed echogenicity, consistent with a large abdominal abscess. Ultrasound guided transcutaneous fine needle aspiration of the abdominal mass was performed 

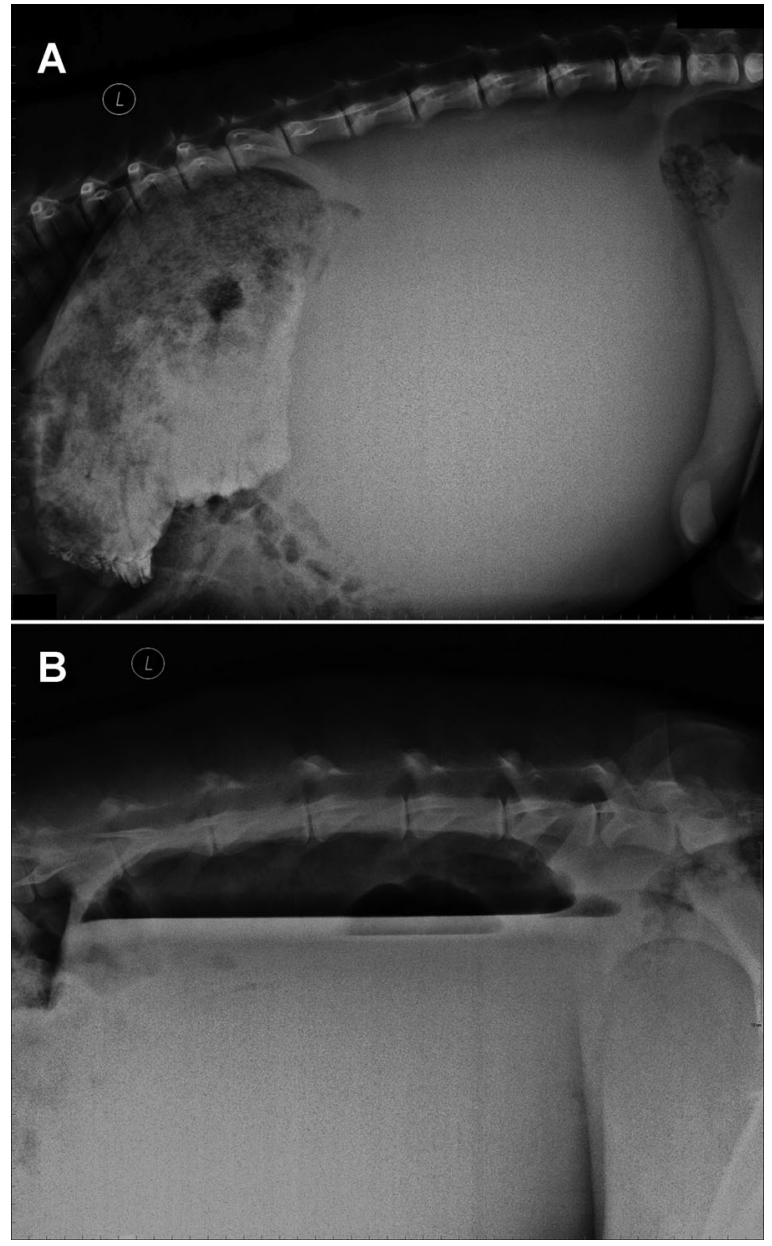

Figure 1 (A) Standing left lateral abdominal radiograph of alpaca 1 showing an extensive soft tissue mass occupying the mid and caudal abdomen, displacing abdominal viscera cranioventrally. (B) Standing left lateral abdominal radiograph of alpaca 2 showing a large fluid-filled abdominal mass with a dorsal gas cap.

with the alpaca in the standing position. The aspirate was thick, white to yellowish, malodorous purulent material. After aerobic and anaerobic bacterial culture, a pure growth of Streptococcus equi ssp. Zooepidemicus was obtained.

Based on the clinical signs, imaging findings, and aspiration results, an exploratory celiotomy was recommended to further define and treat the condition. Preoperatively, flunixin meglumine $(1.1 \mathrm{mg} / \mathrm{kg}$ intravenously [IV]) and ceftiofur $(5 \mathrm{mg} / \mathrm{kg}$ IV) were administered. The alpaca was anesthetized and a $20 \mathrm{~cm}$ ventral median incision was made cranial to the umbilicus. A large mass was identified in the caudal two-thirds of the abdomen with extensive adhesions involving the ventral body wall, gastric compartments, omentum, and small and large intestines. Marsupialization of the mass was considered to be the best and only treatment option. The caudal aspect of the linea alba incision was closed in a simple continuous pattern using 1 polydioxanone, and the cranial aspect $(\sim 5 \mathrm{~cm})$ was left open to perform marsupializa- tion. The external capsule of the abscess was sutured circumferentially to the edges of the linea alba in a simple interrupted pattern using 1 polydioxanone. The subcutaneous tissue and skin were each closed at the caudal aspect of the incision in a simple continuous pattern using 2-0 polydioxanone. The closed portion of the incision was freshly draped to avoid contamination. The abscess was incised and the full thickness of the wall was sutured to the skin edge in a simple continuous pattern using 0 polydioxanone. The alpaca was repositioned into lateral recumbency over the edge of the surgical table and digital manipulation within the abscess capsule allowed for drainage of $\sim 6 \mathrm{~L}$ of purulent material.

Postoperatively, ceftiofur $(8 \mathrm{mg} / \mathrm{kg}$ IV every 12 hours for 6 days), potassium penicillin $(22,000 \mathrm{U} / \mathrm{kg}$ IV every 6 hours for 6 days), and flunixin meglumine $(1.1 \mathrm{mg} / \mathrm{kg}$ IV once daily for 3 days) were administered. Through the marsupialization opening, the internal portion of the abscess was lavaged with $1 \mathrm{~L}$ of saline $(0.9 \% \mathrm{NaCL})$ solution and digitally debrided daily. For the first 3 days after surgery, the abscess was packed with sterile gauze that was changed daily to provide additional debridement. On day 6 , the antibiotic therapy was changed to florfenicol ( $20 \mathrm{mg} / \mathrm{kg}$ subcutaneously every 48 hours for 15 days) based on culture and susceptibility testing results of the abscess fluid. On postoperative day 12 , the abscess produced no further purulent material and the skin sutures were removed. The alpaca was discharged from the hospital 13 days after surgery.

The alpaca was rechecked 20 days after surgery, and the abdominal distension was markedly reduced and there was minimal drainage from the marsupialization site. The abscess was lavaged with saline solution and a small amount of purulent material was digitally debrided. Eight months after surgery, the alpaca was readmitted for evaluation of the reproductive tract. Clinical examination showed the alpaca was in good body condition (BCS 3.5/5) and vital signs were considered normal. There was a $7 \mathrm{~cm}$ reducible abdominal wall hernia palpable at the previous marsupialization site.

Ultrasonographic examination of the abdomen revealed no abnormalities of the gastrointestinal or urogenital tracts, and no evidence of the abscess could be identified. Surgical repair of the hernia was offered but declined by the owner. Two years after surgery, the owner was contacted and reported that the alpaca was doing well. Although the hernia was still present, there were no related complications and she had recently given birth to a healthy cria.

\section{Alpaca 2}

A 5-year-old female Huacaya alpaca was admitted for evaluation of a large intra-abdominal mass first identified by the referring veterinarian while assisting with parturition 2 months before admission. The owner reported that the alpaca had lost a cria from dystocia 2 years previously. In the past month, the alpaca had a poor appetite, lost weight and developed signs of depression. On admission, the alpaca was in fair physical condition (BCS 2.5/5) with a markedly distended, turgid abdomen. She was nursing a healthy cria. Vital signs were within normal limits, and there was no history of pyrexia. 
CBC and serum biochemical profile revealed mild anemia (PCV 22\%; reference interval: 27-45\%), along with a decreased hemoglobin $(8.0 \mathrm{~g} / \mathrm{dL}$; reference interval: $10.55-$ $17.34 \mathrm{~g} / \mathrm{dL})$ and albumin $(2.2 \mathrm{~g} / \mathrm{dL})$. The fibrinogen concentration was increased $(0.7 \mathrm{~g} / \mathrm{dL})$, as was total protein $(7.2 \mathrm{~g} / \mathrm{dL}$; reference interval: $5.4-6.2 \mathrm{~g} / \mathrm{dL})$, globulin $(4.8 \mathrm{~g} / \mathrm{dL})$, and glucose (162 mg/dL; reference interval: $102-153 \mathrm{mg} / \mathrm{dL})$. Standing abdominal radiographs showed a large soft tissue mass with a gas cap dorsally, occupying the caudal $2 / 3$ of the abdomen (Fig 1B). There were 3 fluid lines identified within the mass, suggestive of a multiloculated cavity, most consistent with an abscess. The mass displaced the intestines and gastric compartments cranially. On abdominal ultrasonography, a large ( $>18 \mathrm{~cm}$ in diameter) thick-walled structure containing hypoechoic fluid with diffuse hyperechoic flecks was identified. Ultrasound examination of the reproductive tract per rectum found that the left ovary and left uterine horn were in close proximity to the mass and displaced to the right side of the abdomen. Based on clinical findings, survey radiographs, and abdominal ultrasonography, a presumptive diagnosis of a large abdominal abscess of unknown origin was made and an exploratory celiotomy was recommended.

Preoperatively, lactated Ringer's solution (LRS; 2L bolus IV) and flunixin meglumine $(1.1 \mathrm{mg} / \mathrm{kg}$ IV) were administered. The alpaca was anesthetized and positioned in dorsal recumbency. A $20 \mathrm{~cm}$ ventral median incision was made cranial to the umbilicus, and a large, fully encapsulated intraabdominal mass was identified. Palpation revealed several adhesions between the abscess capsule and the abdominal viscera, including part of the spleen, transverse colon, left ovary, and left horn of the uterus. The abscess occupied most of the left and caudal portions of the abdominal cavity. Aspiration of the mass with a $16 \mathrm{~g}$ needle yielded thick, white, malodorous material, which grew a Gram-negative bacillus on aerobic culture. After collection of the fluid for culture, a dose of potassium penicillin $(22,000 \mathrm{U} / \mathrm{kg})$ and gentamicin $(5 \mathrm{mg} / \mathrm{kg})$ was administered IV.

Because of the extensive adhesions present, a $5 \mathrm{~cm}$ long paramedian incision was created $5 \mathrm{~cm}$ to the left of midline to perform a marsupialization for abscess drainage. The midline incision was closed and protected with a stent bandage and povidone-iodide impregnated plastic sheet (Ioban, 3M Health Care, St. Paul, MN). The abscess capsule was sutured to the cut edges of the rectus abdominis muscle, incorporating the internal and external sheaths, in a Cushing pattern using 0 polyglactin 910 . The alpaca was then tilted $45^{\circ}$ toward the left side and the exposed abscess capsule was incised. Thick, slightly caseous, yellow to white fluid exuded from the opening. The cut edges of the abscess capsule were sutured to the cut edge of the skin with a simple continuous pattern using 2-0 polydioxanone. The alpaca was then positioned in left lateral recumbency, and gentle steady pressure was applied to the caudal abdomen to evacuate as much fluid as possible from the abscess; $\sim 8 \mathrm{~L}$ of thick pus was collected.

Postoperatively, fluids ( 1 L bolus LRS IV every 6 hours for 24 hours), potassium penicillin $(22,000 \mathrm{U} / \mathrm{kg}$ IV every 6hours for 3 days), gentamicin ( $5 \mathrm{mg} / \mathrm{kg}$ IV once daily for 3 days), and flunixin meglumine $(1.1 \mathrm{mg} / \mathrm{kg}$ IV twice daily for 3 days $)$ were administered. The marsupialized abscess was lavaged daily with $2 \mathrm{~L}$ saline for 4 days. On day 3, antibiotic therapy was changed to florfenicol $(20 \mathrm{mg} / \mathrm{kg}$ subcutaneously every 48 hours for 7 days) based on the susceptibility of the Gram-negative bacillus cultured. The alpaca was discharged on day 5 . The owner reported that the marsupialization site was completely healed 3 weeks after surgery.

The alpaca was re-admitted 6 months later for evaluation of an abdominal wall hernia at the marsupialization site. On clinical examination, the alpaca was in good physical condition and vital signs were normal. There was an $8 \times 6 \mathrm{~cm}^{2}$ abdominal wall hernia palpable at the site of previous marsupialization. Most of the hernia was reducible, but an area of thickening and irregularity at the caudal border of the hernial ring could not be reduced. Abdominal ultrasonography confirmed the abdominal wall hernia and revealed a $12 \mathrm{~cm}$ diameter hypoechoic cavity surrounded by a poorly demarcated hyperechoic capsule located in the caudal abdomen, compatible with an abscess.

Based on the previous abdominal abscess and ultrasound findings, a second exploratory celiotomy was recommended. Preoperatively, ceftiofur, potassium penicillin, and flunixin meglumine were administered. The alpaca was anesthetized, and an elliptical skin incision was made around the hernia. Approximately $1 \mathrm{~cm}$ abaxial to the cranial part of the hernial ring, a small incision was made into the abdominal cavity. Digital exploration revealed an $\sim 4 \mathrm{~cm}$ diameter mass immediately adjacent to the body wall. From this mass, a tissue tract was followed $12 \mathrm{~cm}$ deep into the abdomen where a larger (14-16 $\mathrm{cm}^{2}$ diameter) mass was identified. The stalk of tissue connecting the 2 masses was ligated and transected close to the superficial mass. This portion of the stalk and mass were removed en bloc with the hernial sac, ring, and overlying skin. The remaining tissue stalk was used to access the deeper mass. Two liters of thick white to yellow fluid were evacuated from the mass by large-bore needle suction. A fluid sample was submitted for microbial culture and susceptibility testing. The mass was adhered to multiple organs. The body wall incision was closed cranially in 3 layers, leaving the caudal portion open for marsupialization of the abscess. The stalk of the abscess was then sutured to the body wall and skin in a simple continuous pattern using 2-0 polydioxanone. The abscess cavity was thoroughly lavaged with LRS and packed with sterile gauze soaked in a $1 \%$ iodine solution.

Postoperatively, ceftiofur $(5 \mathrm{mg} / \mathrm{kg}$ IV twice daily for 3 days), potassium penicillin (22,000 U/kg IV every 6 hours for 3 days), and flunixin meglumine $(1.1 \mathrm{mg} / \mathrm{kg}$ IV twice daily for 3 days) were administered. The abscess was lavaged with saline and repacked with gauze soaked in $1 \%$ iodine solution every other day. After 5 days, a Pezzer catheter was inserted into the abscess cavity through the marsupialization site to allow continued flushing of the abscess and prevents premature closure of the marsupialization site. Cytology and aerobic culture of the abscess material revealed moderate neutrophils and scant Gram-negative bacilli, which were sensitive to ceftiofur. The alpaca was discharged at 7 days, and the client was instructed to administer ceftiofur $(5 \mathrm{mg} / \mathrm{kg}$ subcutaneously twice daily for 9 days) and lavage the abscess through the Pezzer catheter with $1 \%$ iodine solution once daily for 9 days. 
The alpaca was readmitted 9 days later for a recheck evaluation. The incision site appeared to be healing appropriately and the Pezzer catheter and all sutures were removed. The alpaca was discharged the same day, and the client was instructed to lavage the remaining cavity with $1 \%$ iodine solution using a small red rubber catheter once daily until the catheter no longer fit through the skin incision. The alpaca presented to the hospital 2 years later for a reproductive examination because she had not had a successful pregnancy since surgery. At this time, physical examination was normal and she was in good body condition. A $5 \mathrm{~cm}$ in diameter reducible abdominal wall hernia was present at the previous marsupialization site. The reproductive exam revealed no abnormalities and abdominal ultrasound was unremarkable. Repair of the abdominal wall hernia was offered, but declined.

\section{DISCUSSION}

Although abdominal abscesses in alpacas have not been widely reported, the principles of diagnosis, treatment, and complications are similar to abdominal or umbilical abscesses in other large animal species. However, the clinical presentations noted in the current cases have some interesting differences compared with the typical presentation of other large animal species. $^{1,3,4}$ These alpacas did not have pyrexia and no significant indicators of abscessation, such as leukocytosis and/ or neutrophilia.

The definitive cause of the abscess could not be determined in either alpaca. Bacteria frequently cultured from abscesses in large animals include Streptococcus equi ssp. equi, Strep. equi ssp. zooepidemicus, Fusobacterium necrophorum, and Arcanobacterium pyogenes. ${ }^{4-7}$ Microbial culture of the abscess fluid from alpaca 1 grew Streptococcus equi ssp. zooepidemicus and from alpaca 2, Gram-negative bacilli. Streptococcus equi ssp. zooepidemicus is the causative agent of 'Alpaca Fever,' a severe disease associated with septic pleuritis and peritonitis. ${ }^{8}$ Thus, the culture of Streptococcus equi ssp. zooepidemicus from the abscess of alpaca 1 may demonstrate a previously unrecognized clinical presentation of 'Alpaca Fever' in which bacteria could have invaded the abdominal lymph nodes causing an abdominal abscess, rather than peritonitis. The Gram negative bacilli found in Alpaca 2 may have been intestinal, uterine, or environmental in origin.

Recently, marsupialization has been described to treat a large thoracic abscess in a 6-month-old alpaca, which presented with clinical signs similar to our alpacas, but did have leukocytosis. Another similarity to the cases presented here was that the exact origin of the abscess could not be identified, and the authors hypothesized that hematogenous spread of bacteria through the hemolymphatic system resulted in abscessation of the caudal mediastinal lymph nodes. ${ }^{9}$

Abdominal abscesses are usually well encapsulated and it is important to keep in mind that antibiotic therapy alone will not likely result in complete resolution of the abscess. ${ }^{10}$ Therefore, if en bloc surgical resection of the abscess is not possible because of location or extensive adhesion formation, marsupialization to the body wall should be considered as a treatment option. ${ }^{3}$ Marsupialization can either be performed through a paramedian incision separate from the initial midline incision, as described in alpaca 2, or through a cranial extension of the midline incision, such as the technique used in alpaca 1. ${ }^{3,11}$ Marsupializing the abscess through a smaller, separate incision is thought to help prevent infection of the larger initial incision. $^{10}$

The main complications reported to occur after marsupialization of an abdominal abscess to the ventral body wall in large animals are adhesion formation, herniation, peritonitis, infection of the incision, and recurrence of the abscess. ${ }^{1,3,11,12}$ Complications in our cases included body wall hernia formation in both alpacas, initial incomplete resolution of the abscess in 1 alpaca, and 1 alpaca has not been able to conceive since her procedure. The body wall hernias in both alpacas were at the site of marsupialization, and although the defects were palpable, neither required surgical intervention. Based on previous reports and our experience with these 2 cases, clients should be warned that herniation may be difficult to prevent when marsupializing an abdominal abscess, and that a second procedure may be necessary to correct the hernia.

Finally, the persistence of the abdominal abscess after surgery in alpaca 2 was likely because of premature closure of the marsupialization site, and it resolved completely following a repeat procedure with more attention to aftercare. The lack of conception in this alpaca may be related to the adhesion formation between the abscess and left uterine horn. Despite these complications, surgical marsupialization was successful in resolving the large abdominal abscesses and both alpacas were reportedly healthy 2 years after surgery.

\section{DISCLOSURE}

The authors report no financial or other conflicts related to this report.

\section{REFERENCES}

1. St-Jean G, Anderson DE, Anderson NV, et al: Abdominal pain associated with an umbilical abscess in a llama. Cornell Vet $1993 ; 83: 77-81$

2. Sprake P, Gold JR: Corynebacterium pseudotuberculosis liver abscess in a mature alpaca (Lama pacos). Can Vet J 2012;53:387390

3. Lopez MJ, Markel MD: Umbilical artery marsupialization in a calf. Can Vet J 1996;37:170-171

4. Pusterla N, Whitcomb MB, Wilson WD: Internal abdominal abscesses caused by Streptococcus equi subspecies equi in 10 horses in California between 1989 and 2004. Vet Rec 2007;160:589-592

5. Rumbaugh GE, Smith BP, Carlson GP: Internal abdominal abscesses in the horse: a study of 25 cases. J Am Vet Med Assoc 1978;172:304-309

6. Lechtenberg KF, Nagaraja TG, Leipold HW, et al: Bacteriologic and histologic studies of hepatic abscesses in cattle. Am J Vet Res 1988;49:58-62 
7. Reuss SM, Chaffin MK, Cohen ND: Extrapulmonary disorders associated with Rhodococcus equi infection in foals: 150 cases (1987-2007). J Am Vet Med Assoc 2009;235:855-863

8. Hewson J, Cebra CK: Peritonitis in a llama caused by Streptococcus equi subsp. zooepidemicus. Can Vet J 2001;42:465-467

9. Adolf J, Dykes N, Semevolos S, et al: The diagnosis and treatment of a thoracic abscess in an alpaca. Aust Vet J 2001;79:675-679
10. Hassel DM, Tyler JW, Tucker RL, et al: Clinical vignette: urachal abscess and cystitis in a calf. $J$ Vet Intern Med 1995;9:286-288

11. Steiner A, Lischer CJ, Oertle C: Marsupialization of umbilical vein abscesses with involvement of the liver in 13 calves. Vet Surg 1993;22:184-189

12. Mair TS, Sherlock CE: Surgical drainage and post operative lavage of large abdominal abscesses in six mature horses. Equine Vet J 2011;43(Suppl 39):123-127 